\title{
OPEN Genomic analysis revealed a novel genotype of methicillin-susceptible Staphylococcus aureus isolated from a fatal sepsis case in dengue patient
}

\author{
Soo Tein $\mathrm{Ngoi}^{1 \bowtie}$, Wen Kiong Niek ${ }^{1}$, Yee Wan Lee ${ }^{2}$, Sazaly AbuBakar ${ }^{1,3}$ \& \\ Cindy Shuan Ju Teh ${ }^{1 凶}$
}

Staphylococcus aureus (S. aureus) is an opportunistic pathogen capable of causing serious health implications in susceptible individuals once it invades the host's protective barriers. Methicillinsusceptible $S$. aureus (MSSA) often receives lesser attention although it has been frequently associated with serious infections in human. We aim to investigate the genomic features of a highly virulent yet pan susceptible MSSA strain (coded as HS-MSSA) which caused concurrent bacteraemia in a dengue patient, ultimately resulted in sepsis death of the patient. Whole genome sequence analysis was performed. The draft genome of HS-MSSA is approximately $2.78 \mathrm{Mb}$ (GC content $=32.7 \%$ ) comprising of 2637 predicted coding sequences. In silico genotyping of the HS-MSSA strain revealed a novel combined genotype (t091/ST2990). The HS-MSSA carries a SaPIn1-like pathogenicity island that harbours the staphylococcal enterotoxin and enterotoxin-like genes (sec 3 and selL). The strain-specific $\beta$-lactamase (blaZ)-bearing plasmid region was identified in HS-MSSA. Core genome phylogeny showed that the HS-MSSA strain shared a common ancestry with the European MRSA clone. We report herein the genomic features of an MSSA lineage with novel genotype previously not reported elsewhere.

Staphylococcus aureus (S. aureus) is a Gram-positive round-shaped bacterium commonly found colonizing the skin and mucous membranes of humans. Approximately $15 \%$ of the human population carry $S$. aureus in the nasal cavity, specifically in the anterior nares ${ }^{1}$. S. aureus is an opportunistic pathogen capable of causing serious health implications in susceptible hosts when it enters the bloodstream or internal tissues. Potentially serious infections caused by $S$. aureus include bacteraemia, pneumonia, endocarditis, and osteomyelitis ${ }^{2}$. In fact, the nasal carriage of $S$. aureus has shown a strong association with the aetiology of bacteraemia in susceptible hosts ${ }^{3}$. Bacteraemia caused by invasive $S$. aureus often results in greater mortality rate due to its ability to secrete a wide spectrum of virulence factors that contribute to the evasion of host immune responses ${ }^{4}$.

The severe consequences of $S$. aureus infections are often associated with healthcare facilities due to the breaching of the skin barrier with surgical devices or implants, although it is not uncommon to have community acquired infections ${ }^{5}$. Antimicrobial resistance (AMR) among the healthcare-associated S. aureus infections has been an important global public health issue. Since the emergence of methicillin-resistant S. aureus (MRSA) in the 1960s, this organism has been notorious for its ability to cause severe forms of infection in hospital settings and has eventually spread to the community after 30 years of evolution, most probably due to the transfer of the AMR gene cassette SCCmec to local methicillin-susceptible lineages ${ }^{6}$. This evolutionary theory for the emergence of MRSA lineage has recently been strengthened by whole genome sequence analysis ${ }^{7}$.

Despite receiving less attention compared to its drug-resistant counterpart, the methicillin-susceptible $S$. aureus (MSSA) infection has persisted in both healthcare settings and the community, amidst the increasing global prevalence of $\mathrm{MRSA}^{8}$. In fact, a slow epidemic shift was observed in S. aureus infections worldwide

${ }^{1}$ Department of Medical Microbiology, Faculty of Medicine, University of Malaya, 50603 Kuala Lumpur, Malaysia. ${ }^{2}$ Department of Medicine, Faculty of Medicine, University of Malaya, 50603 Kuala Lumpur, Malaysia. ${ }^{3}$ Tropical Infectious Diseases Research and Education Centre (TIDREC), University of Malaya, 50603 Kuala Lumpur, Malaysia. ${ }^{\circledR}$ email: ngoisootein@um.edu.my; cindysjteh@um.edu.my 
during the past two decades. In the United States (US), multiple clinical studies have recorded an increase in the prevalence of MSSA and a simultaneous decrease in MRSA incidences since $2005^{9,10}$. Recent surveillance in the US has seen an increase in bloodstream infections caused by community acquired MSSA from 2012 to $2017^{11}$. A similar observation was recorded in Europe, whereby a decrease in MRSA bacteraemia was observed since 2005 , accompanied by a continuous increase in MSSA bacteraemia ${ }^{12}$.

Genotyping studies from different geographical regions often revealed a greater genetic diversity among the MSSA populations compared to the MRSA ${ }^{13-15}$. More often than not, invasive MSSA causes greater illness severity in patients suffering from bacteraemia due to its ability to express multiple virulence factors ${ }^{16,17}$. Communityonset MSSA bacteraemia has documented a higher mortality rate compared to that of MRSA in many regions ${ }^{18}$. These observations showed that while much attention has been directed to MRSA infections in recent years, the often neglected MSSA infections have caused a greater and increasing disease burden worldwide.

The ability of the diverse MSSA lineages to express a multitude of virulence genes and the possibility of developing multi-drug resistance phenotypes due to horizontal gene transfer have posed a great risk to public health. Nonetheless, the genetic drives that are responsible for the emergence and persistence of such virulent MSSA lineages remain obscured. In this study, we aimed to investigate the genomic features of an invasive MSSA strain which caused a fatal sepsis case in human. The patient was initially diagnosed with dengue fever upon admission to the hospital, but later developed pneumonia and severe sepsis rapidly and succumbed to multiple organs failure within $24 \mathrm{~h}$ post-admission.

\section{Materials and methods}

Bacterial strain. The community acquired MSSA strain (coded as HS-MSSA) was isolated from the blood culture of a patient in 2017. The clinical presentations as well as the AMR and virulence characteristics related to the infection have been previously described ${ }^{19}$.

Whole genome sequencing, assembly, and annotation. The genomic DNA of the HS-MSSA strain was extracted using DNeasy Blood \& Tissue Kit (Qiagen, Hilden, Germany) and the quality of the extracted genomic DNA was evaluated based on spectrophotometric measurements ${ }^{20}$. The HS-MSSA genomic DNA was subsequently subjected to next-generation sequencing via Illumina Genome Analyzer $_{\text {IIx }}$ (GA2x, pipeline version 1.80 ) by a commercial sequencing vendor. Paired-end $2 \times 150$ bp reads were generated using Illumina chemistry. The quality of the sequence reads was assessed and assembled de novo using the CLC Genomics Workbench version 5.1 (CLC Bio, Aarhus, Denmark) with default parameters. Contigs with an average coverage of 30 and above were selected to represent the bacterial draft genome for further analyses. Reordering of the HS-MSSA contigs was achieved via genomic mapping with the reference genome $S$. aureus subspecies aureus NCTC 8325 (National Center for Biotechnology Information (NCBI) GenBank accession number: NC007795) by using Mauve software version $2.4 .0^{21}$. The subset of the contigs that were mapped to the complete chromosome of the S. aureus NCTC 8325 was identified as the chromosomal region of the HS-MSSA strain. Genomic annotation was performed using the webserver Rapid Annotations using Subsystems Technology (RAST; http://rast.nmpdr $. \mathrm{org} /)^{22}$. The viewing and preliminary sequence-based comparison between the annotated HS-MSSA and reference genomes were done using SEED Viewer version 2.0 (http://pubseed.theseed.org/ $)^{23}$, and further validated by using Mauve.

Whole genome sequence analyses. The chromosomal region of the HS-MSSA was subjected to a microbial genome search using the NCBI Basic Local Alignment Search Tool (BLAST; https://blast.ncbi.nlm. nih.gov/Blast.cgi) to identify genetically similar S. aureus strains (taxonomy ID: 46170) from the complete and draft genomes database curated in the NCBI GenBank. The BLAST search was performed using the discontinuous megablast algorithm with default parameters. Multiple genomes alignment and visualization were performed using Mauve and BLAST Ring Image Generator (BRIG) software ${ }^{24}$. The genotype of the HS-MSSA strain was inferred in-silico by using web-based servers. The conventional seven-gene multi-locus sequence typing (MLST), whole genome MLST (wgMLST), and ribosomal MLST (rMLST) predictions made use of the S. aureus database in PubMLST (https://pubmlst.org/saureus/) sited at the University of Oxford ${ }^{25}$. The online analysis tools available from the Center for Genomic Epidemiology (CGE) server (http://www.genomicepidemiology. org/) were used to predict the staphylococcal protein A (spa) type, presence of SCCmec elements, plasmid elements, and pathogenicity genes in the HS-MSSA genome ${ }^{26-29}$. Phage-associated genes and genomic regions in the HS-MSSA genome was identified using PHAge Search Tool Enhanced Release server (PHASTER; http:// phaster.ca/) ${ }^{30,31}$. The genomic islands were predicted using IslandViewer 4 (http://www.pathogenomics.sfu.ca/ islandviewer $/ 2^{32}$. All genomic predictions were completed using default parameters, validated by cross-examination with RAST-annotation output, and manually interrogated using NCBI BLAST nucleotide search tool.

AMR genes prediction. The online tool, Resistance Genes Identifier version 5.1.0 (RGI; https://card.mcmas ter.ca/analyze/rgi) was used to identify AMR genes in the assembled HS-MSSA genome ${ }^{33}$. The AMR genes selection criteria were set to perfect (100\% identity) and strict ( $>95 \%$ identity) hits only to the curated reference sequences in the Comprehensive Antibiotic Resistance Database (CARD; https://card.mcmaster.ca/). Prediction of partial genes was accepted as the HS-MSSA genome examined in this study resembles an incomplete genome. The RGI predictions were cross-checked with ResFinder version 3.2 (https://cge.cbs.dtu.dk/services/ ResFinder/). AMR genes prediction results obtained using default parameters ${ }^{34}$. All predicted AMR genes were validated by cross-examination with RAST-annotation output and manually interrogated using NCBI BLAST. 


\begin{tabular}{|l|l|l|l|}
\hline & Whole genome & Chromosomal region & Other contigs \\
\hline Genome size (bp) & $2,776,811$ & $2,740,810$ & 36,001 \\
\hline Number of contigs & 52 & 37 & 15 \\
\hline GC content (\%) & 32.7 & 32.7 & 34 \\
\hline N50 (bp) & 109,723 & 106,828 & 19,763 \\
\hline Number of subsystems & 289 & 286 & 5 \\
\hline Number of coding sequences (CDSs) & 2637 & 2606 & 31 \\
\hline Number of unique CDSs ${ }^{\mathrm{a}}$ & 222 & 195 & 27 \\
\hline Number of RNAs & 65 & 60 & 5 \\
\hline
\end{tabular}

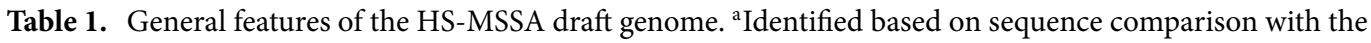
NCTC8325 reference genome. Unique CDSs are present only in the HS-MSSA draft genome.

Core genome single-nucleotide polymorphisms-based phylogenetic analysis. The online tool, Reference sequence Alignment based Phylogeny builder version 1.12 (REALPHY; https://realphy.unibas.ch/ realphy/) was used to identify relevant single nucleotide polymorphism (SNP) sites for core genome phylogenetic analysis ${ }^{35}$. Complete and draft genomes of 20 global S. aureus strains were selected for core genome SNPbased (cgSNP) phylogenetic analysis based on considerations including high genetic identity (from microbial genome BLAST analysis), geographical distribution, type of organisms (MSSA and MRSA), and MLST clonal complexes. The selected S. aureus genomes were retrieved from the NCBI GenBank and PubMLST databases and the strains information is tabulated and appended as Supplementary Table S1. SNP calling was performed using default input parameters and S. aureus NCTC 8325 was used as the reference genome for multiple alignments. The multiple genomes sequence alignment generated by the REALPHY tool was subsequently used to construct a phylogenetic tree. An unrooted tree was inferred via the approximate maximum likelihood (ML) method using FastTree2 software ${ }^{36}$, and visualized using FigTree version 1.4.3 (https://github.com/rambaut/figtr ee/releases) ${ }^{37} .100$ bootstrap replicates were performed to support the phylogenetic inferences made in this study for practical reasons. A bootstrap analysis with 100-500 replicates is sufficient to produce qualitatively comparable support values with more than $99.5 \%$ correlation with the reference values in ML trees with higher numbers of bootstrap replicates ${ }^{38}$.

7-loci MLST-based phylogenetic analysis. The concatenated allelic sequences of the seven housekeeping genes $(\operatorname{arcC}, \operatorname{aro\mathrm {E}}, g l p \mathrm{~F}, g m k, p t a, t p i$, and $y q i \mathrm{~L}$ ) for the central STs of the major clonal complexes (CC1, CC5, CC8, CC15, and CC97) and other frequently encountered clonal complexes (CC22, CC30, CC45, and CC93) were used to construct a phylogenetic tree. Multiple alignments of the concatenated sequences were performed by Molecular Evolutionary Genetics Analysis (MEGA) X ${ }^{39}$. Next, the aligned sequences were subjected to a statistical selection of best-fit phylogenetic model using jModelTest version 2.1.6 $6^{40}$. An unrooted tree was subsequently inferred via the ML method using MEGA X software.

\section{Results and discussion}

Genomic features of HS-MSSA strain. The size of the HS-MSSA draft genome is approximately $2.78 \mathrm{Mb}$ (GC content $=32.7 \%$ ), of which $2,740,810$ bp belongs to the genomic region that corresponds to the chromosome of S. aureus NCTC 8325. A total of 2,637 coding sequences (CDSs) and 65 RNAs were predicted in the HSMSSA draft genome. The general features of the HS-MSSA draft genome are summarized in Table 1. Majority of the total predicted CDSs are shared between HS-MSSA and the reference genome NCTC $8325(n=2,415)$, with a percentage identity that ranged from 31 to $100 \%$ (median $=99.75 \%$ ). A total of 222 CDSs were found unique to the HS-MSSA genome, mainly encoding for hypothetical proteins, phage proteins, mobile genetic elements, efflux pumps, virulence and resistance factors. This Whole Genome Shotgun project has been deposited at the NCBI GenBank under the accession number VCMW00000000. The version described in this paper is version VCMW01000000.

Genomic comparison revealed a great genetic resemblance between HS-MSSA strain and MRSA clones. Out of the total 52 contigs generated from de novo assembly, 37 of these are associated with S. aureus chromosomal regions. Microbial genome BLAST analysis showed that the HS-MSSA chromosome shares greatest genetic homology ( $97 \%$ query coverage with $99.25 \%$ nucleotide identity at $\mathrm{E}$ value $=0$ ) with the MRSA strains isolated in the United States of America (USA). More than half of the MRSA strains that are genetically similar to the HS-MSSA were obtained from a clonal cluster of community acquired MRSA that caused skin and soft tissue infections (SSTIs) in a military base in the USA ${ }^{41}$. Similar genetic homology was observed between the MRSA USA300 clones and HS-MSSA, albeit at slightly lower scores. The two USA300 clones that showed greatest genetic homology with HS-MSSA are USA300_TCH1516 (NC_010079) and USA300_SUR1 (NZ_CP009423), both being community acquired MRSA strains isolated from USA and Republic of Suriname in South America respectively. A circular genome map was constructed to illustrate the genetic resemblance of HS-MSSA to the representative MRSA clones from the North and South America (MRSA strains 2148. C01 (NZ_CP017094) and USA300_SUR1), and an MSSA strain KT/314250 (NZ_AOCP00000000) isolated in 


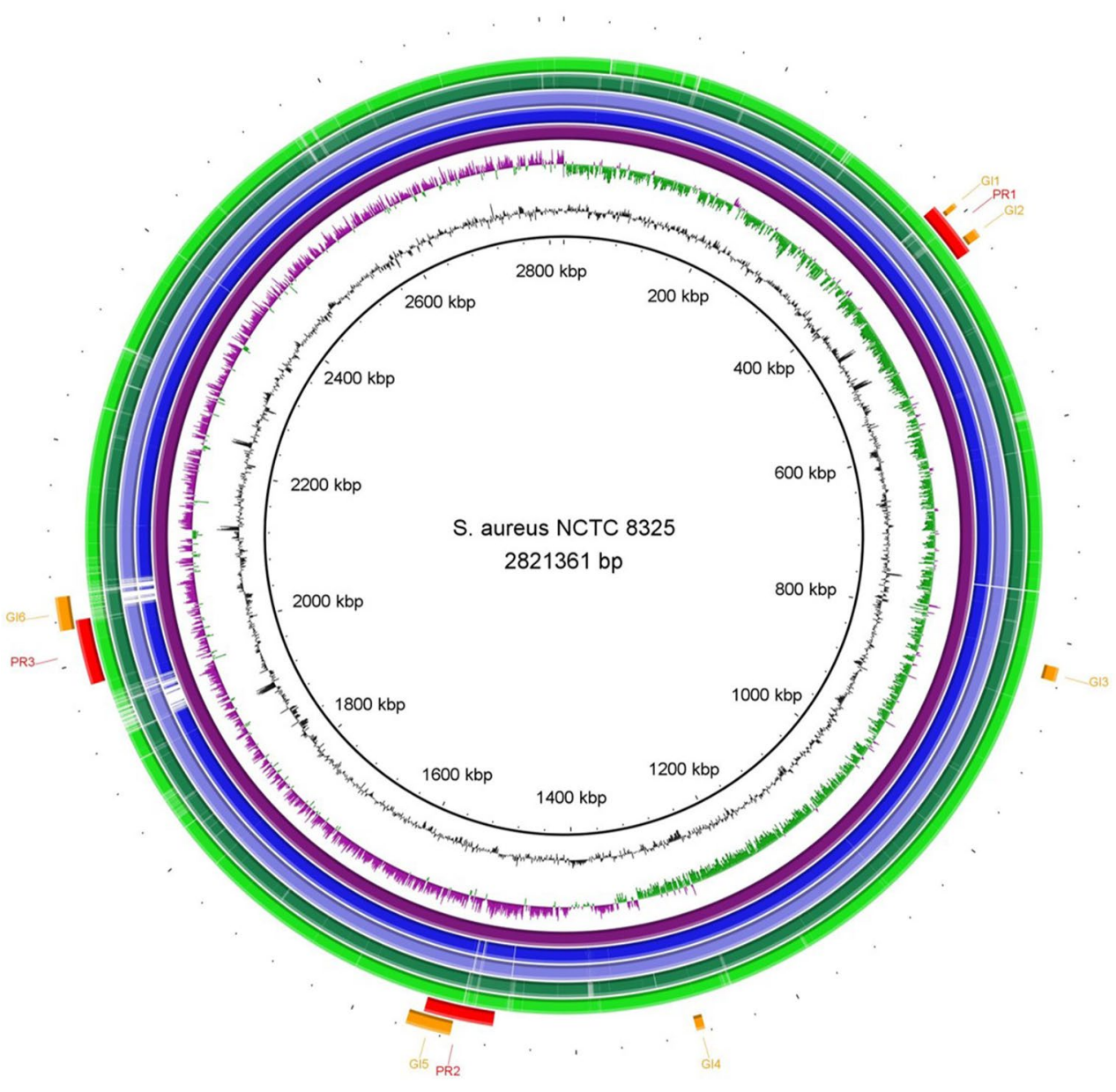

GC content

GC skew
GC skew(-)
GC skew(+)

S. aureus NCTC 8325

$100 \%$ identity

$70 \%$ identity

$50 \%$ identity

MRSA strain 2148.C01

$100 \%$ identity

$70 \%$ identity

$50 \%$ identity

MRSA strain USA300_SUR1

$100 \%$ identity

$70 \%$ identity

$50 \%$ identity

MSSA strain KT314250

$100 \%$ identity

$70 \%$ identity

$50 \%$ identity

HS-MSSA

$100 \%$ identity

$70 \%$ identity

$50 \%$ identity

Phage regions

Genomic islands

Figure 1. Genome map of the representative MRSA and MSSA strains. The genome map was generated using BLAST Ring Image Generator (BRIG) ${ }^{24}$. The genomes of two MSSA strains isolated in Malaysia (HS-MSSA and KT/314,250) and two community acquired MRSA clones (2148.C01 and USA300_SUR1 isolated in the North and South America respectively) were mapped to the reference genome S. aureus NCTC 8325. From the inner to the outermost ring, the first (innermost) ring shows the genome size in kbp, followed by GC skew (purple and green), GC content (black), S. aureus NCTC 8325 (purple), MRSA strain 2148.C01 (dark blue), MRSA strain USA300_SUR1 (light blue), MSSA strain KT/314,250 (dark green), and HS-MSSA strain (light green). The predicted phage regions (red) and genomic islands (orange) in the HS-MSSA genome are shown in arcs.

Malaysia (Fig. 1). The HS-MSSA strain was seen sharing more common regions of genetic variation with the MRSA clones than with the local MSSA strain at different sites on the genome.

The great genomic resemblance between the HS-MSSA strain and the MRSA clones from the American continent indicates the possible common ancestry between these lineages, despite the wide geographical distance where the strains were originated. The MRSA clones originated from the USA that showed high genetic homology with HS-MSSA were all community acquired, capable of causing SSTIs and sepsis, and were largely resistant to chlorhexidine ${ }^{41-44}$. The South American MRSA strain (USA300_SUR1) that caused an outbreak of SSTIs shared common MLST type (ST8) with the community acquired USA300 clone from Texas, USA ${ }^{43}$. The complete genome analysis revealed that the South American outbreak strains were genetic variants of the North American USA300 clone ${ }^{45}$. Previous genomic studies have proven that USA300 clones are clonally related to the European nosocomial MRSA strain COL (NC_002951) emerged from a methicillin-susceptible ancestral strain via the acquisition of methicillin resistance determinant following the introduction of methicillin in $1959^{43,46}$. Therefore, the MRSA clones USA300 and COL are most likely sharing a common MSSA ancestry but evolved separately through different MRSA lineages ${ }^{47}$. Accordingly, the close genomic resemblance of the HS-MSSA strain to these MRSA clones suggests that this MSSA lineage in Malaysia could have been the less evolved (with reference to AMR) descendant of the common MSSA ancestry with the MRSA clones in the USA and Europe. This inference was made on the basis that chromosomal recombination occurs in S. aureus at low frequencies, except for regions associated with virulence and pathogenicity ${ }^{48}$. The descendants of this MSSA ancestral strain have spread widely over different continents and persisted in the human populations over the years, causing community acquired SSTIs and invasive infections in the susceptible hosts. 
Presence of strain-specific blaZ-bearing plasmid in HS-MSSA. Detailed examination of the 15 contigs that were not included in the HS-MSSA chromosomal region revealed a 19,763 bp region (contig-33) identified as a plasmid-associated genomic region. BLAST analysis of contig-33 revealed the closest plasmid homolog as pl1_M2024 (CP047022), at 100\% query coverage with 99\% nucleotide identity (E-value =0). The pl1_M2024 is a complete plasmid retrieved from the nosocomial MRSA clone with spa type t304 and MLST type ST6 (clone t304/ST6) which caused recurrent outbreaks in a hospital in Denmark since 2011 $1^{49}$. This MRSA clone t304/ST6 was detected and predominated among the MRSA strains isolated in a teaching hospital in Oman within the same period ${ }^{50}$. However, this clone remains rarely reported outside these two geographical regions. The finding of similar plasmids in the HS-MSSA strain in Malaysia and MRSA clone in European region suggests possible horizontal gene transfer event, which could have occurred due to chance events after the divergence from the common MSSA ancestral strain. One other hypothesis is that the HS-MSSA strain could have evolved from an MSSA lineage carrying this plasmid, of which some of its descendants might have diverged to another evolutionary path via the acquisition of SCCmec gene cassette. The latter hypothesis is more plausible as this plasmid carries the penicillin-resistant blaZ gene. Based on an earlier study using Staphylococcus species mainly from the bovine origin, the authors demonstrated that plasmid-borne blaZ is often species-specific and lack conjugative transfer mechanisms, thus making horizontal gene transfer an extremely rare event $t^{51}$. Therefore, the presence of this blaZ-bearing plasmid in the HS-MSSA strain supports our inference on the common ancestry of this local MSSA with the MRSA clones in European regions.

In silico genotyping of HS-MSSA revealed novel S. aureus genotype t091/ST2990. In silico MLST analyses showed that HS-MSSA belongs to the sequence type ST2990, with its identity confirmed as S. aureus by rMLST. The 7-loci allelic profile for HS-MSSA is 1-1-1-1-330-1-10, arranged in the order arcC-aroEglpF-gmk-pta-tpi-yqiL. All loci MLST (wgMLST) results showed 1358 exact matches to sequence type ST2990 (Supplementary Table S2). This sequence type (ST) belongs to MSLT clonal complex CC1. The S. aureus strain 128A deposited in the database (PubMLST ID: 5521) shares the same sequence type as HS-MSSA and was also isolated in Malaysia, earlier in 2014. S. aureus 128A was isolated from the infected wound of an elderly diabetic patient (66 years old) examined in a tertiary hospital in the capital Kuala Lumpur. The strain was found susceptible to oxacillin, methicillin, and vancomycin upon investigation. The S. aureus $128 \mathrm{~A}$ was confirmed as community acquired MSSA which causes wound infection. The spa type of HS-MSSA is t091 (spa repeat succession 07-23-21-17-34-12-23-02-12-23). Therefore, the combined genotype of the HS-MSSA strain is noted as t091/ ST2990 (spa type/MLST type).

Similar to the S. aureus strain 128A which shares the identical sequence type (ST2990), the HS-MSSA strain examined in this study was isolated from a diabetic patient who was admitted to the hospital due to dengue fever ${ }^{19}$. However, instead of causing SSTI (infected diabetic wound) as strain 128A did, the HS-MSSA was more invasive and caused a bloodstream infection that resulted in severe sepsis. The common STs of S. aureus in Malaysia, especially the most studied MRSA strains, mainly comprised of the pandemic clone ST239, with ST22 clone being increasingly documented in the past $\operatorname{decade}^{52-54}$. Similar to the global epidemiology of MSSA, the few studies that examined the MSSA genotypes in Malaysia documented a higher genetic diversity compared to the MRSA populations in this region ${ }^{55-57}$. MRSA with spa type 091 has been reported in Malaysia, but S. aureus strain with genotype t091/ST2990 has not been previously documented ${ }^{58}$. Nonetheless, the isolation of ST2990 strains from different geographical regions suggests that this MSSA genotype may already be widely disseminated in Malaysia. Although S. aureus of CC1 complex (including ST1, ST188, ST2990, etc.) does not predominate in Malaysia, the persistence of these genotypes throughout the years may indicate their evolutionary success ${ }^{55,57,58}$.

\begin{abstract}
Absence of staphylococcal chromosome cassette mec elements throughout the evolutionary process of HS-MSSA. The staphylococcal chromosome cassette mec (SCCmec) elements were not detected in the HS-MSSA genome based on SCCmecFinder analysis at $90 \%$ identity threshold and a minimum length of $60 \%$. The absence of SCCmec elements in the HS-MSSA genome is reflected in the methicillin-susceptible phenotype of this strain. The SCCmec genomic cassette is a mobile genomic region with two important gene complexes, mec-gene complex encoding methicillin resistance and cassette chromosome recombinase (ccr)-gene complex encoding site-specific recombinase(s) for the mobility of this DNA region ${ }^{59}$. The presence of SCCmec region in the bacterial chromosome is a signature of MRSA strains and the acquisition of which results in the emergence of new MRSA lineage from an ancestral MSSA strain.

While the acquisition of SCCmec elements provides a selective advantage to $S$. aureus, it is not uncommon for it to spontaneously excised from the MRSA in human hosts ${ }^{60}$. Partial excision is sufficient to result in methicillinsusceptible phenotype, and most often such MSSA strains showed great genetic homology to its MRSA counterpart, although less prevalent ${ }^{60,61}$. Given the genomic resemblance of the HS-MSSA strain to the global MRSA clones, we decided to investigate whether such an excision event has occurred in this local MSSA strain. In order to identify possible remnants of SCCmec cassette or its homologs in the genome, the database search was repeated at $60 \%$ identity threshold with $30 \%$ minimum query cover length. The search result remains the same, i.e. intact or partial mec genes and the whole cassette was not detected, but a $1.5 \mathrm{~kb}$ DNA region associated with the insertion sequence-like IS1272 in SCCmec type I was detected in contig-4, albeit with considerable genetic variation (94\% query coverage and $82 \%$ nucleotide identity). BLAST analysis of this $1.5 \mathrm{~kb}$ region revealed the presence of a pseudogene of IS1182 family transposase in S. aureus (CP039848; 98\% query coverage, 99\% nucleotide identity, E value $=0$ ). In short, none of the SCCmec elements was detected except for the $1.5 \mathrm{~kb}$ DNA region homologous to IS1272 commonly found in the mec-gene complex in MRSA that may also present infrequently in MSSA ${ }^{62}$.
\end{abstract}




\begin{tabular}{|l|l|l|l|l|l|l|}
\hline AMR gene & Contig & Region position (bp) & SNP & Genomic region & Drug class & AMR gene family \\
\hline arlS & 7 & $73,606-74,961$ & - & Chromosome & Fluoroquinolone antibiotic; acridine dye & $\begin{array}{l}\text { Major facilitator superfamily (MFS) antibiotic } \\
\text { efflux pump }\end{array}$ \\
\hline arlR & 7 & $74,958-75,617$ & - & Chromosome & Fluoroquinolone antibiotic; acridine dye & $\begin{array}{l}\text { Major facilitator superfamily (MFS) antibiotic } \\
\text { efflux pump }\end{array}$ \\
\hline norA & 16 & $13,813-14,979$ & - & Chromosome & Fluoroquinolone antibiotic & $\begin{array}{l}\text { Major facilitator superfamily (MFS) antibiotic } \\
\text { efflux pump }\end{array}$ \\
\hline mgrA & 16 & $22,630-23,073$ & - & Chromosome & $\begin{array}{l}\text { Fluoroquinolone antibiotic; cephalosporin; } \\
\text { penam; tetracycline antibiotic; peptide antibi- } \\
\text { otic; acridine dye }\end{array}$ & $\begin{array}{l}\text { ATP-binding cassette (ABC) antibiotic efflux } \\
\text { pump; major facilitator superfamily (MFS) } \\
\text { antibiotic efflux pump }\end{array}$ \\
\hline ImrS & 21 & $40,760-42,205$ & - & Chromosome & $\begin{array}{l}\text { Macrolide antibiotic; aminoglycoside antibiotic; } \\
\text { oxazolidinone antibiotic; diaminopyrimidine } \\
\text { antibiotic; phenicol antibiotic }\end{array}$ & $\begin{array}{l}\text { Major facilitator superfamily (MFS) antibiotic } \\
\text { efflux pump }\end{array}$ \\
\hline mepR & 32 & $38,133-38,552$ & - & Chromosome & Glycylcycline; tetracycline antibiotic & $\begin{array}{l}\text { Multidrug and toxic compound extrusion } \\
\text { (MATE) transporter }\end{array}$ \\
\hline mepA & 32 & $38,659-40,014$ & - & Chromosome & Glycylcycline; tetracycline antibiotic & $\begin{array}{l}\text { Multidrug and toxic compound extrusion } \\
\text { (MATE) transporter }\end{array}$ \\
\hline blaZ & 33 & $4979-5824$ & - & Plasmid & Penam & blaZ beta-lactamase \\
\hline tet(38) & 43 & $1800-3158$ & - & Chromosome & Tetracycline antibiotic & $\begin{array}{l}\text { Major facilitator superfamily (MFS) antibiotic } \\
\text { efflux pump }\end{array}$ \\
\hline GlpT variant & 32 & $40,641-41,999$ & A100V & Chromosome & Fosfomycin & GlpT \\
\hline
\end{tabular}

Table 2. Antimicrobial resistance-conferring genes identified in the HS-MSSA genome.

Pathogenicity analysis of HS-MSSA revealed limited antimicrobial resistance-conferring genes but multiple virulence factors. The HS-MSSA strain was found susceptible to amoxicillin-clavulanate, cefoxitin, ceftriaxone, ciprofloxacin, clindamycin, cloxacillin, erythromycin, fusidic acid, gentamicin, penicillin-G, piperacillin-tazobactam, rifampicin, and sulfamethoxazole-trimethoprim ${ }^{19}$. Genomic sequence analysis revealed a low number of AMR-conferring genes compared to virulence genes, and a majority of which are involved in bacterial efflux systems (Table 2). Partial genes were not identified in the HS-MSSA genome. The pan-susceptibility of the HS-MSSA strain to antimicrobial agents is reflected in its genomic composition. Although multiple efflux pumps and its regulatory systems involve in antimicrobial agents and biocides resistance are present in the HS-MSSA genome, it is not surprising to observe a susceptible phenotype in the strain. Similar to the Gram-negative bacteria, efflux pumps in Gram-positive organisms often confer only low-level resistance and are only effective when overexpressed due to accumulation of mutations in response to environmental stresses ${ }^{63}$. A recent study has reported an interesting phenomenon, whereby the prevalence of certain efflux pump genes was found to be associated with the genotypes of S. aureus ${ }^{64}$. The presence of norA and mepA genes in the HS-MSSA strain is typical of S. aureus lineages in the Asian region, while norB or $m d e \mathrm{~A}$ is common among European S. aureus ${ }^{64}$. Unlike plasmid-borne genes, these efflux pump genes are chromosomally located hence is fairly conserved within the S. aureus lineages. The close genetic proximity of the HS-MSSA strain carrying norA and mepA genes to the European MRSA lineages suggests that the divergence of these two S. aureus lineages might have occurred early in the evolutionary timeline, followed by local adaptations in the different geographical regions.

The pathogenicity genes analysis showed that the HS-MSSA harbours 536 CDSs which matched to known S. aureus pathogenicity families (with 59\% uncharacterized or hypothetical proteins). Cross-examination with virulence genes analysis showed that the HS-MSSA strain carries multiple genes encoding for bacterial adherence, enzyme production, host immune evasion, various prominent staphylococcal toxins, a type VII secretion system, and intact regulatory operons for virulence expression (global accessory gene regulator operon, agrABCD; staphylococcal accessory regulator, sar). The virulence analysis of the HS-MSSA strain is reported in detail in our earlier publication ${ }^{19}$ (Supplementary Table S3). Majority of the prominent toxin-producing genes are found associated with the highly variable genomic regions in the HS-MSSA genome. The HS-MSSA resembles a community acquired, highly virulent but pan-susceptible $S$. aureus strain that was capable of causing an invasive bloodstream infection in the human host that resulted in fatal sepsis. It is a common notion that the MSSA lineages that cause human infections often harbour a wider array of virulence genes compared to the MRSA $^{16}$. The combined effect of multiple virulent factors present in the MSSA genomes has resulted in greater severity of infection, especially one that causes invasive infection ${ }^{17}$. This notion is experimentally proven in a study that examined the transcriptional changes in an MRSA strain when exposed to different antibiotics ${ }^{65}$. In this study, Choe and colleagues proved that the expression of the $\beta$-lactam resistance (Bla/Mec system) in the MRSA represses the transcription of the agr operon, thus halting the production of the virulence factors that are regulated by this operon. Therefore, the ability of the HS-MSSA to cause severe sepsis in the human host could be related to the lack of SCCmec genes cassette in its genome, thus the expression of its multiple virulence factors was not impeded.

HS-MSSA harbours multiple genomic islands associated with pathogenicity and prophages. Three prophage regions were identified in the HS-MSSA chromosome. Phage region 1 and 3 (PR1 and PR3) are incomplete prophages, while phage region 2 (PR2) is an intact prophage spanning a $64.1 \mathrm{~kb}$ 


\begin{tabular}{|c|c|c|c|c|c|c|c|}
\hline Region & Region length (kb) & Region position $^{\mathrm{a}}$ (bp) & Ordered contig numbers & GC $\%$ & PHASTER annotation & Phage identity $(\%)$ & PHASTER prediction $^{c}$ \\
\hline PR1 & 51.5 & $379,258-430,819$ & 13 & 31.6 & $\begin{array}{l}\text { PHAGE_Staphy_PT1028_ } \\
\text { NC_007045 }\end{array}$ & 9 & incomplete \\
\hline PR2 & 64.1 & $1,475,209-1,539,349$ & 15,4 & 32.5 & $\begin{array}{l}\text { PHAGE_Staphy_phi2958PVL_ } \\
\text { NC_011344 }\end{array}$ & 40 & intact \\
\hline PR3 & 60.1 & $1,978,784-2,038,945$ & 11 & 31.8 & $\begin{array}{l}\text { PHAGE_Staphy_phiN315_ } \\
\text { NC_004740 }\end{array}$ & 52 & incomplete \\
\hline
\end{tabular}

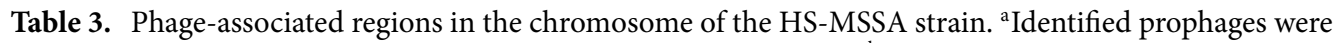
over multiple contigs that were concatenated for PHASTER analysis. ${ }^{b}$ Phage identity was calculated based on the percentage of phage-associated genes over the total protein-coding sequences identified in the phage region. ${ }^{\mathrm{c}} \mathrm{A}$ prediction of whether the phage region contains genes necessary for lysogeny.

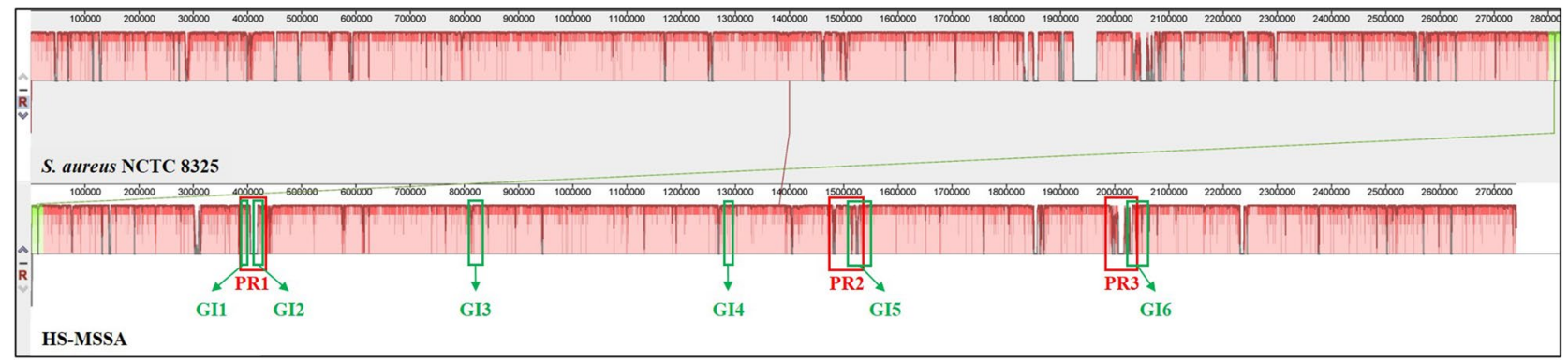

Figure 2. The genomic alignment of HS-MSSA chromosome against the reference genome S. aureus NCTC 8325. The similarity plot was generated using Mauve version $2.4 .0^{21}$. Genomic regions with a low percentage of similarity are seen as gaps in the similarity plot of the respective genomes. The relative positions of the phageassociated regions (denoted as PR1 to PR3) and the genomic islands (denoted as GI1 to GI6) on the HS-MSSA chromosome are highlighted in red and green boxes respectively.

\begin{tabular}{|c|c|c|c|c|}
\hline Region & Region position $^{\mathrm{a}}$ (bp) & Region length (bp) & Ordered contig numbers & Gene products \\
\hline GI1 & $388,058-392,685$ & 4627 & 13 & Ribosomal proteins; DNA-binding proteins; SaPI proteins \\
\hline GI2 & $416,923-425,593$ & 8670 & 13 & SaPI proteins; phage proteins; enterotoxins (SEC3, SElL) \\
\hline GI3 & $822,496-835,323$ & 12,827 & 30,2 & $\begin{array}{l}\text { Fibrinogen-binding protein (ClfA); extracellular and plasma-binding protein; staphylococcal } \\
\text { adhesins; thermonuclease; cold-shock protein }\end{array}$ \\
\hline GI4 & $1,285,799-1,292,852$ & 7053 & 27 & Hypothetical proteins \\
\hline GI5 & $1,511,627-1,551,821$ & 40,194 & 4 & Phage proteins \\
\hline GI6 & $2,031,470-2,061,814$ & 30,344 & 11 & Phage proteins \\
\hline
\end{tabular}

Table 4. Genomic islands identified in the HS-MSSA chromosome. ${ }^{a}$ Identified genomic islands were over multiple contigs that were concatenated for IslandViewer4 analysis.

genomic region (Table 3). The PR1 was identified on contig-13 of the HS-MSSA genome which mainly comprised of staphylococcal toxin-production genes that encode for exotoxins and the superantigen enterotoxins (SEC3 and SElL). Genome mapping showed that this genomic region is not present in the staphylococcal reference genome NCTC 8325, thus resembling a genomic insertion (Fig. 2). The intact prophage PR2 was located on contig-4 and found associated with genes encoding for staphylococcal Panton-Valentine leukocidin (lukFS-PV). When mapped to the reference genome $S$. aureus NCTC 8325, the PR2 showed great genetic similarity with a $72 \mathrm{~kb}$ prophage on NCTC 8325 . The PR3 was identified on contig-11, closely associated with genes encoding for $\beta$-hemolysin $(h l b)$, cytolytic pore-forming protein (lukGH), extracellular adherence protein (eap), and staphylococcal complement inhibitor $(s c n)$. A total of six genomic islands (GI1-GI6) were identified in the HS-MSSA chromosome by using S. aureus NCTC 8325 as the reference (Fig. 2). The genomic size, contigs involved, and the types of genes identified in the genomic islands (GIs) are listed in Table 4. Generally, the regions encoding GI1GI2, GI5, and GI6 correspond with the phage regions PR1, PR2, and PR3, respectively. The genomic region that spanned GI1 and GI2 resembles a staphylococcal pathogenicity island (SaPI) harbouring the enterotoxin-encoding genes sec3 and selL similar to the SaPIn1 in the MRSA strain N315 (BA000018, previously AP003129) ${ }^{66,67}$. An array of staphylococcal virulence genes mainly associated with bacterial adherence and stress response were found in GI4, while GI5 consisted of mainly hypothetical proteins.

Majority of the genomic islands identified in the HS-MSSA genome are associated with bacterial virulence and pathogenicity. Genomic islands are specific chromosomal DNA regions that include genes conferring a survival advantage to the bacterial host, such as virulence and resistance ${ }^{59}$. The association of the genomic islands, prophages, and SaPI observed in the HS-MSSA genome is not uncommon as these genetic entities are often 


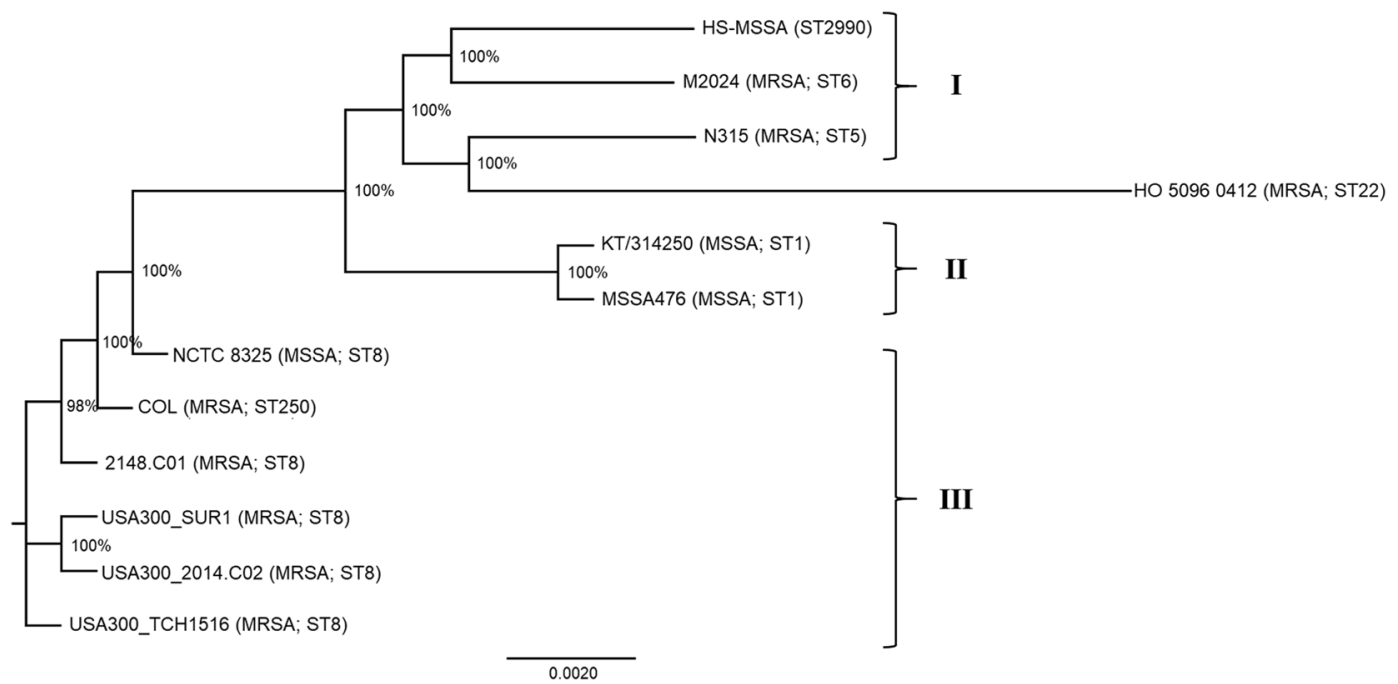

Figure 3. Phylogenomic tree inferred by maximum-likelihood method from SNP-based core genomes alignment of the $S$. aureus strains. The multiple genome sequence alignments of 11 global $S$. aureus strains were generated by the online tool REALPHY using S. aureus NCTC 8325 as the reference genome. An unrooted phylogenomic tree was inferred by approximately Maximum Likelihood method using Generalized TimeReversible (GTR) model with gamma distribution of rates and 100 bootstrap replicates. Bootstrap support value is indicated as a percentage at each node. The roman numerals denote the arbitrary clusters of strains. The ST22 reference genome HO 50960412 (NC_017763) resembles a phylogenetic outgroup.

interrelated in S. aureus. Pathogenicity islands are genomic islands that carry virulence factors and are mobilized by helper phages ${ }^{66}$. Moreover, prophages and pathogenicity islands are mobile elements in the staphylococcal chromosome that constitutes the genetic diversity among different $S$. aureus lineages. The prevalence of specific staphylococcal prophages was shown associated with the clonal background of $S$. aureus, i.e. different lineages may harbour different types of prophage ${ }^{68}$. Meanwhile, the SaPIs actively involved in the pathogenic evolution of $S$. aureus via the lateral transfer of virulence genes ${ }^{69}$, hence a greater genetic homology in SaPIs may indicate a closer phylogenetic relationship. Therefore, the sharing of genetically similar SaPIs between HS-MSSA and MRSA N315, and common phage regions between HS-MSSA and NCTC 8325 suggest a clonal relationship, or more probably, the sharing of a recent common ancestor that acquired the common phage or SaPI regions prior to evolutionary divergence.

Phylogenetic analysis revealed common ancestry of HS-MSSA with MRSA clones originated from European regions. Representative S. aureus complete genomes were selected for cgSNP-based phylogenetic analysis to elucidate the probable evolutionary origin of HS-MSSA strain. The reference genomes were selected based on greatest nucleotide identity in BLAST analysis (MRSA strain 2148.C01 and USA300 clones), sharing of similar mobile genomic regions (M2024, NCTC 8325 and N315), same geographical origin (KT/314250), same clonal complex (MSSA476), and representative MSSA and MRSA genomes (MSSA476 and COL) from European region (potential common ancestry). The HS-MSSA genome showed $\geq 94 \%$ genome coverage and $\geq 99.16 \%$ nucleotide identity (E value $=0$ ) with all selected $S$. aureus genomes. The ST22 reference genome HO 50960412 (NC_017763) from a clonal complex (CC22) less closely related to other S. aureus strains was used as antgroup in constructing the ML tree.

The phylogenetic analysis results revealed that the HS-MSSA strain formed a distinctive clade by itself, sharing a more recent common ancestry with the MRSA clones in Europe and Japan (M2024 and N315) (Fig. 3). The MRSA strain N315 isolated from the pharyngeal smear of a Japanese patient in 1982 belongs to the clonotype II-A which is prevalent in Japan and USA ${ }^{67}$. Together with another MRSA strain M2024 (NZ_CP047021) which caused recurrent clonal outbreaks in Denmark ${ }^{49}$, the three $S$. aureus strains formed a loose cluster with relatively divergent evolutionary paths and geographical origins (Cluster I). Interestingly, the MSSA476 from the United Kingdom that shares a common clonal complex (CC1) with HS-MSSA formed another distinctive cluster (II) although both are community acquired MSSA capable of causing bacteraemia in human hosts and carried the strain-specific plasmid-borne blaZ gene ${ }^{70}$. Similarly, the MSSA strain KT/314250 which was also isolated in Malaysia formed a separate clade distinctive from the HS-MSSA lineage but is tightly clustered with the MSSA476 strain. The representative S. aureus strains from Europe (NCTC 8325 and COL) and MRSA clones from America formed a third cluster (III) more distantly related to the HS-MSSA strain.

The phylogenetic relationships of the HS-MSSA strain with the global S. aureus strains as predicted by the core genome SNPs analysis supported our inference on the HS-MSSA lineage. The sharing of a relatively recent common ancestry with the European-originated MRSA clone M2024 suggests the existence of an ancestral MSSA strain which, in turn, shares a common ancestry with the MSSA476 lineage in Europe. The descendants of this MSSA lineage had since taken separate evolutionary paths, developing antimicrobial resistance or virulence as an 


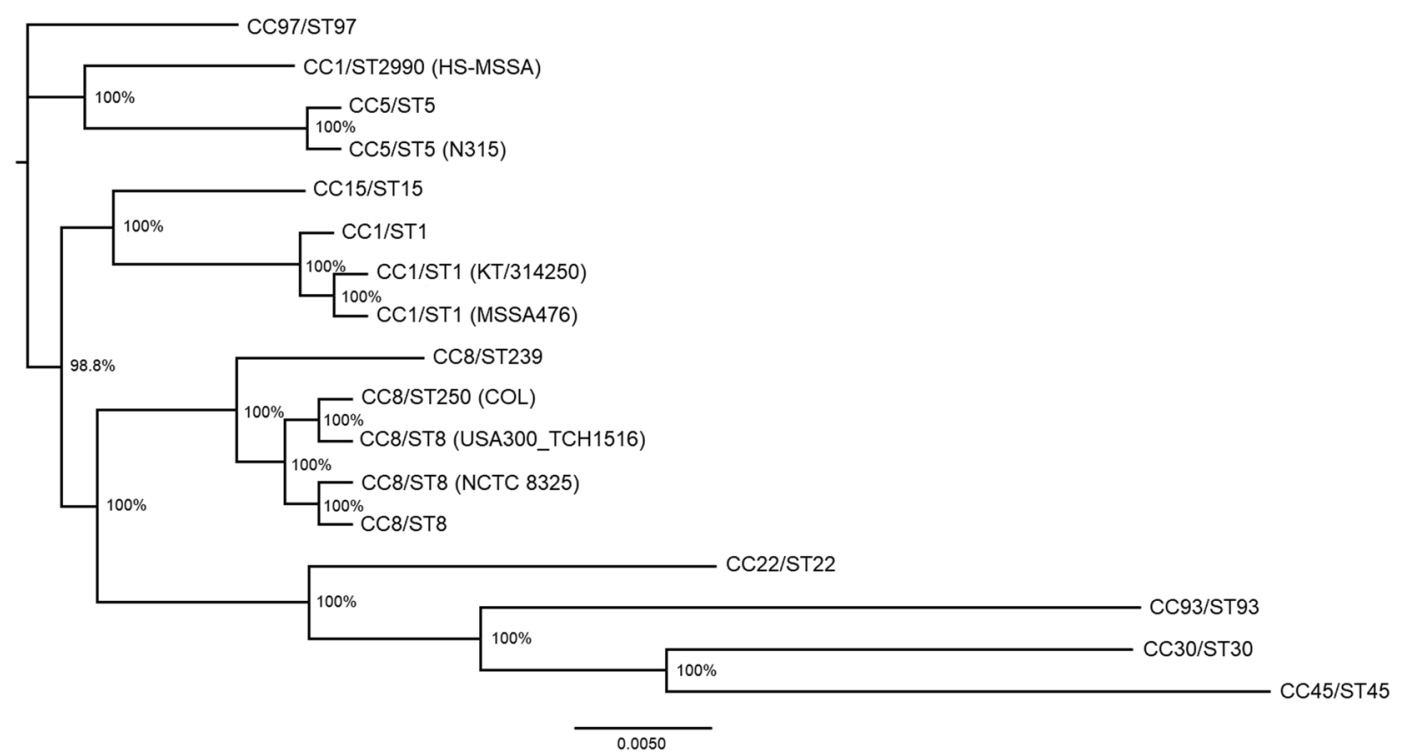

Figure 4. Phylogenomic tree inferred by maximum-likelihood method from SNP-based core genomes alignment of the $S$. aureus strains representing different multi-locus sequence typing (MLST) clonal complexes. The multiple genome sequence alignments of $17 \mathrm{~S}$. aureus strains were generated by the online tool REALPHY using S. aureus NCTC 8325 as the reference genome. An unrooted phylogenomic tree was inferred by approximately Maximum Likelihood method using Generalized Time-Reversible (GTR) model with gamma distribution rates and 100 bootstrap replicates. Bootstrap support value is indicated as a percentage at each node. The representative genomes for each clonal complex are denoted as clonal complex/sequence type (i.e. CC1/ST1 represents MLST sequence type 1 in clonal complex 1). The PubMLST ID and European Nucleotide Archive (ENA) reference numbers of the representative genomes are available in Supplementary Table S1.

adaptive feature in different settings. The relatively more distant genetic relationship between the HS-MSSA and KT/314250, both isolated in Malaysia, infers that multiple MSSA lineages exist and persisted in this geographical region. This observation is not unexpected, given that previous epidemiological studies in Malaysia often reached the same conclusion that the MSSA populations in Malaysia are genetically diverse ${ }^{55-57}$. Unlike most of the MSSA strains that have circulated in this region, the MSSA strain 128A that shares a common MLST genotype (ST2990) with HS-MSSA only appeared on scientific records in 2014. Based on the limited strain data available in the PubMLST database for the MSSA strain 128A, we hypothesize that the ST2990 strains in Malaysia showed variable clinical manifestations, ranging from non-invasive SSTI to severe bacteraemia. The HS-MSSA lineage has probably accumulated sufficient adaptive features through the acquisition of mobile genetic elements, giving rise to its unusual virulence in causing severe invasive infection in human host ${ }^{19}$. Moreover, the close genetic proximity with the MRSA clones and its genome plasticity also suggests that the HS-MSSA lineage could provide a stable genetic environment to allow the acquisition of genetic elements that confer drug resistance ${ }^{6}$. Therefore, continuous molecular surveillance to detect highly virulent MSSA in clinical settings is essential to allow timely management of invasive staphylococcal infections and the development of potential anti-virulence therapy.

HS-MSSA (CC1/ST2990) showed a closer phylogenetic relationship with CC5 than CC1 based on cgSNP analysis. Upon inspection of the MLST clonal complexes of the global S. aureus strains selected for cgSNP-based phylogenetic analysis, we observed that the HS-MSSA strain genotyped as CC1/ST2990 was not found on the same clade as other CC1 strains. In order to investigate the accuracy of this phylogenetic clustering, representative genomes of the central STs for the major CCs were retrieved from the PubMLST database for further analysis ${ }^{71}$. Overall, the clustering of the STs corresponded with the CC grouping based on the 7-loci MLST scheme (Fig. 4). However, the HS-MSSA formed a distinctive clade sharing a common node with the CC5/ST5 clade (100\% bootstrap support) instead of being grouped in the CC1 cluster. On the contrary, the phylogenetic analysis based on the concatenated sequences of the representative STs revealed that the HS-MSSA indeed showed a closer phylogenetic relationship with CC1/ST1 and CC8/ST8 (92\% bootstrap support) (Supplementary Fig. S1). Nonetheless, the multiple genomes alignment of HS-MSSA and the representative CC1/ ST1 genomes MSSA476 and ERR410082 (PubMLST ID: 5965) showed that multiple genomic insertions were observed in both variable (PR1-PR3) and conserved regions of the HS-MSSA genome (Supplementary Fig. S2). This could have contributed to the greater phylogenetic divergent of HS-MSSA from other CC1 strains based on cgSNP analysis.

Our observations support the previous notion that the current grouping of S. aureus strains into clonal complexes based on the 7-loci MLST scheme requires reconsideration to better discriminate the strains ${ }^{71}$. This observation is not unexpected as cgSNP-based analysis infers the phylogenetic relationship between organisms based on thousands of core genes, hence providing a greater resolution than the 7-loci MLST that examines a relatively small portion of the genome. Nonetheless, current advancement in next-generation sequencing 
technology has improved the resolution of MLST-based phylogeny. The core genome MLST (cgMLST) which involves the assessment of 1351 loci (for S. aureus) and the whole genome MLST (wgMLST) are the more appropriate approaches for inferring phylogenetic relationship of genetically clonal strains. Previous studies using different bacterial species have observed a high concordance between cgMLST and wgMLST with SNP-based analysis, generating phylogenetic trees with great statistical similarity and almost equivalent clustering of bacterial strains ${ }^{72,73}$. Therefore, the current clustering of ST2990 into CC1 needs to be re-assessed by the scientific community. Nonetheless, more ST2990 genomes should be included for cgSNP-based analysis in the future to verify this notion. The present study is limited by only one ST2990 (HS-MSSA) genome that was available on publicly accessible genomic databases at the time of writing.

\section{Conclusion}

We report herein a novel genotype of MSSA (t091/ST2990) of community origin that caused severe sepsis in the human host. Upon examination, the HS-MSSA genome harbours only very few AMR genes, which was reflected in its pan-susceptible phenotype. The HS-MSSA strain carries multiple virulence genes in its genome, including prominent staphylococcal toxin genes that are associated with mobile genetic elements (prophages and SaPI). The HS-MSSA strain shares a common ancestry with the MRSA clone originated from the European region, but instead of developing drug resistance, it has evolved through a different path via the acquisition of multiple virulence and pathogenicity factors. Due to the limited epidemiological data available for this novel genotype (t091/ST2990), we could not conclude that this MSSA lineage has emerged locally or the HS-MSSA strain was just a sporadic strain imported from other regions without molecular typing data. Although the patient reported no history of recent travels upon admission, we cannot rule out the possibility of local transmission. Therefore, increased effort in molecular surveillance is essential to allow the development of appropriate treatment strategies to reduce the mortality risk of severe invasive infections caused by this organism.

\section{Data availability}

All data generated or analysed during this study are included in this published article. The genomic data of the HS-MSSA strain generated and analysed during the current study are available in the NCBI GenBank database at https://www.ncbi.nlm.nih.gov/genome/, under the accession VCMW00000000.

Received: 18 August 2020; Accepted: 28 January 2021

Published online: 01 March 2021

\section{References}

1. Taylor, T. A. \& Unakal, C. G. Staphylococcus aureus (StatPearls Publishing, Treasure Island, 2018).

2. Tong, S. Y. C., Davis, J. S., Eichenberger, E., Holland, T. L. \& Fowler, V. G. Staphylococcus aureus infections: Epidemiology, pathophysiology, clinical manifestations, and management. Clin. Microbiol. Rev. 28, 603-661 (2015).

3. von Eiff, C., Becker, K., Machka, K., Stammer, H. \& Peters, G. Nasal carriage as a source of Staphylococcus aureus bacteremia. N. Engl. J. Med. 344, 11-16 (2001).

4. Thomer, L., Schneewind, O. \& Missiakas, D. Pathogenesis of Staphylococcus aureus bloodstream infections. Annu. Rev. Pathol. 11, 343-364 (2016).

5. Fetsch, A. E. Staphylococcus aureus (Academic Press, Cambridge, 2018).

6. Deurenberg, R. H. \& Stobberingh, E. E. The evolution of Staphylococcus aureus. Infect. Genet. Evol. 8, 747-763 (2008).

7. Weterings, V. et al. Next-generation sequence analysis reveals transfer of methicillin resistance to a methicillin-susceptible Staphylococcus aureus strain that subsequently caused a methicillin-resistant Staphylococcus aureus outbreak: A descriptive study. J. Clin. Microbiol. 55, 2808-2816 (2017).

8. Mostofsky, E., Lipsitch, M. \& Regev-Yochay, G. Is methicillin-resistant Staphylococcus aureus replacing methicillin-susceptible $S$. aureus?. J. Antimicrob. Chemothr. 66, 2199-2214 (2011).

9. Acree, M. E., Morgan, E. \& David, M. Z. S. aureus infections in Chicago, 2006-2014: Increase in CA MSSA and decrease in MRSA incidence. Infect. Cont. Hosp. Ep. 38, 1226-1234 (2017).

10. Sutter, D. E. et al. Changing susceptibility of Staphylococcus aureus in a US pediatric population. Pediatrics 137, e20153099. https ://doi.org/10.1542/peds.2015-3099 (2016).

11. Kourtis, A. P. et al. Vital signs: Epidemiology and recent trends in methicillin-resistant and in methicillin-susceptible Staphylococcus aureus bloodstream infections - United States. MMWR Morb. Mortal. Wkly. Rep. 68, 214-219 (2019).

12. de Kraker, M. E. A. et al. The changing epidemiology of bacteraemias in Europe: Trends from the European Antimicrobial Resistance Surveillance System. Clin. Microbiol. Infect. 19, 860-868 (2013).

13. Grundmann, H. et al. The dynamic changes of dominant clones of Staphylococcus aureus causing bloodstream infections in the European region: Results of a second structured survey. Eurosurveillance 19, 20987. https://doi.org/10.2807/1560-7917.ES201 4.19.49.20987 (2014)

14. Ho, C.-M. et al. Concomitant genotyping revealed diverse spreading between methicillin-resistant Staphylococcus aureus and methicillin-susceptible Staphylococcus aureus in central Taiwan. J. Microbiol. Immunol. 49, 363-370 (2016).

15. Aung, M. S. et al. Drug resistance and genetic characteristics of clinical isolates of staphylococci in Myanmar: High prevalence of PVL among methicillin-susceptible Staphylococcus aureus belonging to various sequence types. New Microb. New Infect. 10, 58-65 (2016).

16. Bride, L. D. L. et al. Differences in resistance profiles and virulence genes among methicillin-resistant and methicillin-susceptible Staphylococcus aureus of different lineages at a public tertiary hospital. Rev. Soc. Bras. Med. Trop. https://doi.org/10.1590/00378682-0095-2019 (2019).

17. Wehrhahn, M. C. et al. Illness severity in community-onset invasive Staphylococcus aureus infection and the presence of virulence genes. J. Infect. Dis. 205, 1840-1848 (2012).

18. Tom, S. et al. Case fatality ratio and mortality rate trends of community-onset Staphylococcus aureus bacteraemia. Clin. Microbiol. Infect. 20, 630-632 (2014).

19. Ngoi, S. T. et al. Atypical presentation of methicillin-susceptible Staphylococcus aureus infection in a dengue-positive patient: A case report with virulence genes analysis. Pathog. 9, 190. https://doi.org/10.3390/pathogens9030190 (2020).

20. Psifidi, A. et al. Comparison of eleven methods for genomic DNA extraction suitable for large-scale whole-genome genotyping and long-term DNA banking using blood samples. PLOS ONE 10, e0115960. https://doi.org/10.1371/journal.pone.0115960 (2015). 
21. Darling, A. E., Mau, B. \& Perna, N. T. progressiveMauve: Multiple genome alignment with gene gain, loss and rearrangement. PLoS ONE 5, e11147. https://doi.org/10.1371/journal.pone.0011147 (2010).

22. Aziz, R. K. et al. The RAST Server: Rapid annotations using subsystems technology. BMC Genom. 9, 75. https://doi. org/10.1186/1471-2164-9-75 (2008).

23. Overbeek, R. et al. The SEED and the Rapid Annotation of microbial genomes using Subsystems Technology (RAST). Nucleic Acids Res. 42, D206-D214. https://doi.org/10.1093/nar/gkt1226 (2014).

24. Alikhan, N.-F., Petty, N. K., Ben Zakour, N. L. \& Beatson, S. A. BLAST Ring Image Generator (BRIG): Simple prokaryote genome comparisons. BMC Genom. 12, 402. https://doi.org/10.1186/1471-2164-12-402 (2011).

25. Jolley, K. A., Bray, J. E. \& Maiden, M. C. J. Open-access bacterial population genomics: BIGSdb software, the PubMLST.org website and their applications. Wellcome Open Res. 3, 124. https://doi.org/10.12688/wellcomeopenres.14826.1 (2018).

26. Bartels, M. D. et al. Comparing whole-genome sequencing with Sanger sequencing for spa typing of methicillin-resistant Staphylococcus aureus. J. Clin. Microbiol. 52, 4305-4308 (2014).

27. Cosentino, S., Voldby Larsen, M., Møller Aarestrup, F. \& Lund, O. PathogenFinder: Distinguishing friend from foe using bacterial whole genome sequence data. PLoS ONE 8, e77302. https://doi.org/10.1371/journal.pone.0077302 (2013).

28. Carattoli, A. et al. In silico detection and typing of plasmids using PlasmidFinder and plasmid multilocus sequence typing. Antimicrob. Agents Ch. 58, 3895-3903 (2014).

29. Kaya, H. et al. SCCmecFinder, a web-based tool for typing of staphylococcal cassette chromosome mec in Staphylococcus aureus using whole-genome sequence data. $m$ Sphere 3, e00612-00617. https://doi.org/10.1128/mSphere.00612-17 (2018).

30. Arndt, D. et al. PHASTER: A better, faster version of the PHAST phage search tool. Nucleic Acids Res. 44, W16-W21. https://doi. org/10.1093/nar/gkw387 (2016).

31. Zhou, Y., Liang, Y., Lynch, K. H., Dennis, J. J. \& Wishart, D. S. PHAST: A fast phage search tool. Nucleic Acids Res. 39, W347-W352. https://doi.org/10.1093/nar/gkr485 (2011).

32. Bertelli, C. et al. IslandViewer 4: Expanded prediction of genomic islands for larger-scale datasets. Nucleic Acids Res. 45, 30-35 (2017).

33. Alcock, B. P. et al. CARD 2020: Antibiotic resistome surveillance with the comprehensive antibiotic resistance database. Nucleic Acids Res. 48, D517-D525. https://doi.org/10.1093/nar/gkz935 (2020).

34. Zankari, E. et al. Identification of acquired antimicrobial resistance genes. J. Antimicrob. Chemoth. 67, 2640-2644 (2012).

35. Bertels, F., Silander, O. K., Pachkov, M., Rainey, P. B. \& van Nimwegen, E. Automated reconstruction of whole-genome phylogenies from short-sequence reads. Mol. Biol. Evol. 31, 1077-1088 (2014).

36. Price, M. N., Dehal, P. S. \& Arkin, A. P. FastTree 2: Approximately maximum-likelihood trees for large alignments. PLoS ONE 5, e9490. https://doi.org/10.1371/journal.pone.0009490 (2010).

37. Rambaut, A. FigTree v1.4.3, http://tree.bio.ed.ac.uk/software/figtree/ (2016).

38. Pattengale, N. D., Alipour, M., Bininda-Emonds, O. R. P., Moret, B. M. E. \& Stamatakis, A. How many bootstrap replicates are necessary?. J. Comput. Biol. 17, 337-354 (2010).

39. Kumar, S., Stecher, G., Li, M., Knyaz, C. \& Tamura, K. MEGA X: Molecular evolutionary genetics analysis across computing platforms. Mol. Biol. Evol. 35, 1547-1549 (2018).

40. Darriba, D., Taboada, G. L., Doallo, R. \& Posada, D. jModelTest 2: More models, new heuristics and parallel computing. Nat. Methods 9, 772-772 (2012).

41. LaBreck, P. T. et al. Conjugative transfer of a novel staphylococcal plasmid encoding the biocide resistance gene, qacA. Front. Microbiol. 9, 1-17 (2018).

42. Schlett, C. D. et al. Prevalence of chlorhexidine-resistant methicillin-resistant Staphylococcus aureus following prolonged exposure. Antimicrob. Agents Ch. 58, 4404-4410 (2014).

43. Highlander, S. K. et al. Subtle genetic changes enhance virulence of methicillin resistant and sensitive Staphylococcus aureus. BMC Microbiol. 7, 99. https://doi.org/10.1186/1471-2180-7-99 (2007).

44. Johnson, R. C. et al. Recurrent methicillin-resistant Staphylococcus aureus cutaneous abscesses and selection of reduced chlorhexidine susceptibility during chlorhexidine use. J. Clin. Microbiol. 53, 3677-3682 (2015).

45. Sabat, A. J. et al. Complete-genome sequencing elucidates outbreak dynamics of CA-MRSA USA300 (ST8-spa t008) in an academic hospital of Paramaribo, Republic of Suriname. Sci. Rep. 7, 41050. https://doi.org/10.1038/srep41050 (2017).

46. Baba, T., Bae, T., Schneewind, O., Takeuchi, F. \& Hiramatsu, K. Genome sequence of Staphylococcus aureus strain Newman and comparative analysis of staphylococcal genomes: Polymorphism and evolution of two major pathogenicity islands. J. Bacteriol. 190, 300-310 (2008).

47. Robinson, D. A. \& Enright, M. C. Evolutionary models of the emergence of methicillin-resistant Staphylococcus aureus. Antimicrob. Agents Ch. 47, 3926-3934 (2003).

48. Feng, Y. et al. Evolution and pathogenesis of Staphylococcus aureus: Lessons learned from genotyping and comparative genomics. FEMS Microbiol. Rev. 32, 23-37 (2008).

49. Bartels, M. D. et al. Monitoring meticillin resistant Staphylococcus aureus and its spread in Copenhagen, Denmark, 2013, through routine whole genome sequencing. Eurosurveillance 20, 21112. https://doi.org/10.2807/1560-7917.ES2015.20.17.21112 (2015).

50. Udo, E. E., Al-Lawati, B. A. H., Al-Muharmi, Z. \& Thukral, S. S. Genotyping of methicillin-resistant Staphylococcus aureus in the Sultan Qaboos University Hospital, Oman reveals the dominance of Panton-Valentine leucocidin-negative ST6-IV/t304 clone. New Microb. New Infect. 2, 100-105 (2014).

51. Olsen, J. E., Christensen, H. \& Aarestrup, F. M. Diversity and evolution of blaZ from Staphylococcus aureus and coagulase-negative staphylococci. J. Antimicrob. Chemoth. 57, 450-460 (2006).

52. Sit, P. S. et al. Prevalence of methicillin-resistant Staphylococcus aureus (MRSA) infection and the molecular characteristics of MRSA bacteraemia over a two-year period in a tertiary teaching hospital in Malaysia. BMC Infect. Dis. 17, 274. https://doi.org/10.1186/ s12879-017-2384-y (2017).

53. Lim, K. T., Hanifah, Y. A., Mohd Yusof, M. Y., Ito, T. \& Thong, K. L. Comparison of methicillin-resistant Staphylococcus aureus strains isolated in 2003 and 2008 with an emergence of multidrug resistant ST22: SCCmec IV clone in a tertiary hospital, Malaysia. J. Microbiol. Immunol. 46, 224-233 (2013).

54. Lim, K. T., Hanifah, Y. A., Yusof, M. Y. M., Goering, R. V. \& Thong, K. L. Temporal changes in the genotypes of methicillin-resistant Staphylococcus aureus strains isolated from a tertiary Malaysian hospital based on MLST, spa, and mec-associated dru typing. Diagn. Microb. Infect. Dis. 74, 106-112 (2012).

55. Ghasemzadeh-Moghaddam, H. et al. Methicillin-susceptible Staphylococcus aureus from clinical and community sources are genetically diverse. Int. J. Med. Microbiol. 301, 347-353 (2011).

56. Ghasemzadeh-Moghaddam, H., Neela, V., Goering, R. \& Mariana, N. Methicillin sensitive Staphylococcus aureus (MSSA) isolates as a potential source for the emergence of USA 300 methicillin resistant Staphylococcus aureus (MRSA) in Malaysia. Trop. Biomed. 29, 429-433 (2012).

57. Lim, K. T., Yeo, C. C., Suhaili, Z. \& Thong, K. L. Comparison of methicillin-resistant and methicillin-sensitive Staphylococcus aureus strains isolated from a tertiary hospital in Terengganu, Malaysia. Jpn. J. Infect. Dis. 65, 502-509 (2012).

58. Ghaznavi-Rad, E. et al. Predominance and emergence of clones of hospital-acquired methicillin-resistant Staphylococcus aureus in Malaysia. J. Clin. Microbiol. 48, 867-872 (2010).

59. Hiramatsu, K. et al. Genomic basis for methicillin resistance in Staphylococcus aureus. Infect. Chemother. 45, 117-136 (2013). 
60. Boundy, S. et al. Spontaneous staphylococcal cassette chromosome mec element excision in Staphylococcus aureus nasal carriers. J. Clin. Microbiol. 50, 469-471 (2012).

61. Donnio, P.-Y. et al. Partial excision of the chromosomal cassette containing the methicillin resistance determinant results in methicillin-susceptible Staphylococcus aureus. J. Clin. Microbiol. 43, 4191-4193 (2005).

62. Kobayashi, N., Urasawa, S., Uehara, N. \& Watanabe, N. Distribution of insertion sequence-like element IS1272 and its position relative to methicillin resistance genes in clinically important staphylococci. Antimicrob. Agents Ch. 43, 2780-2782 (1999).

63. Schindler, B. D. \& Kaatz, G. W. Multidrug efflux pumps of Gram-positive bacteria. Drug Resist. Update. 27, 1-13 (2016).

64. Hassanzadeh, S., Ganjloo, S., Pourmand, M. R., Mashhadi, R. \& Ghazvini, K. Epidemiology of efflux pumps genes mediating resistance among Staphylococcus aureus: A systematic review. Microb. Pathogenesis 139, 103850. https://doi.org/10.1016/j.micpa th.2019.103850 (2020).

65. Choe, D. et al. Genome-scale analysis of methicillin-resistant Staphylococcus aureus USA300 reveals a tradeoff between pathogenesis and drug resistance. Sci. Rep. 8, 2215. https://doi.org/10.1038/s41598-018-20661-1 (2018).

66. Novick, R. P. \& Ram, G. Staphylococcal pathogenicity islands-movers and shakers in the genomic firmament. Curr. Opin. Microbiol. 38, 197-204 (2017).

67. Kuroda, M. et al. Whole genome sequencing of meticillin-resistant Staphylococcus aureus. The Lancet 357, 1225-1240 (2001).

68. Goerke, C. et al. Diversity of prophages in dominant Staphylococcus aureus clonal lineages. J. Bacteriol. 191, 3462-3468 (2009).

69. Sato', Y., Omoe, K., Ono, H. K., Nakane, A. \& Hu, D. L. A novel comprehensive analysis method for Staphylococcus aureus pathogenicity islands. Microbiol. Immunol. 57, 91-99 (2013).

70. Holden, M. T. G. et al. Complete genomes of two clinical Staphylococcus aureus strains: Evidence for the rapid evolution of virulence and drug resistance. P. Natl. Acad. Sci. USA 101, 9786-9791 (2004).

71. Dabul, A. N. G. \& Camargo, I. L. B. C. Clonal complexes of Staphylococcus aureus: All mixed and together. FEMS Microbiol. Lett. 351, 7-8 (2014).

72. Henri, C. et al. An assessment of different genomic approaches for inferring phylogeny of Listeria monocytogenes. Front. Microbiol. 8, 1-13 (2017).

73. Pearce, M. E. et al. Comparative analysis of core genome MLST and SNP typing within a European Salmonella serovar Enteritidis outbreak. Int. J. Food Microbiol. 274, 1-11 (2018).

\section{Acknowledgements}

This study is funded by the University of Malaya Research Fund, RU008-2018.

\section{Author contributions}

S.T.N. analysed and interpreted the genomic data and wrote the manuscript. Y.W.L. analysed and interpreted the patient data. W.K.N. extracted genomic DNA and performed a quality check of the DNA. W.K.N. and S.A.B. contributed to the genomic data analyses. C.S.J.T. provided laboratory support, analysed and interpreted the data. All authors contributed to critically reviewing and revising the manuscript. All authors read and approved the final version of the manuscript.

\section{Competing interests}

The authors declare no competing interests.

\section{Additional information}

Supplementary Information The online version contains supplementary material available at https://doi. org/10.1038/s41598-021-83661-8.

Correspondence and requests for materials should be addressed to S.T.N. or C.S.J.T.

Reprints and permissions information is available at www.nature.com/reprints.

Publisher's note Springer Nature remains neutral with regard to jurisdictional claims in published maps and institutional affiliations.

(c) (i) Open Access This article is licensed under a Creative Commons Attribution 4.0 International (c) License, which permits use, sharing, adaptation, distribution and reproduction in any medium or format, as long as you give appropriate credit to the original author(s) and the source, provide a link to the Creative Commons licence, and indicate if changes were made. The images or other third party material in this article are included in the article's Creative Commons licence, unless indicated otherwise in a credit line to the material. If material is not included in the article's Creative Commons licence and your intended use is not permitted by statutory regulation or exceeds the permitted use, you will need to obtain permission directly from the copyright holder. To view a copy of this licence, visit http://creativecommons.org/licenses/by/4.0/.

(C) The Author(s) 2021 